$\begin{array}{ll}\text { le portiQue } & \text { Le Portique } \\ \text { Revue de philosophie et de sciences humaines }\end{array}$

$8 \mid 2001$

Nietzsche et le divin

\title{
Nietzsche et l'avenir de la religion
}

\section{Paul Valadier sj}

\section{OpenEdition}

\section{Journals}

\section{Édition électronique}

URL : http://journals.openedition.org/leportique/199

DOI : 10.4000/leportique.199

ISSN : $1777-5280$

\section{Éditeur}

Association "Les Amis du Portique"

Édition imprimée

Date de publication : 1 septembre 2001

ISSN : 1283-8594

\section{Référence électronique}

Paul Valadier sj, « Nietzsche et l'avenir de la religion », Le Portique [En ligne], 8 | 2001, mis en ligne le 09 mars 2005, consulté le 12 avril 2021. URL : http://journals.openedition.org/leportique/199 ; DOI : https://doi.org/10.4000/leportique.199

Ce document a été généré automatiquement le 12 avril 2021.

Tous droits réservés 


\title{
Nietzsche et l'avenir de la religion
}

\author{
Paul Valadier sj
}

1 Nietzsche, qui définit l'homme comme «fabricateur de dieux» est, avant tout, un critique de l'idolâtrie qui peut prendre bien d'autres formes que celle de la religion. Le christianisme est, selon lui, à l'origine de sa propre «euthanasie » qui résulte d'une contradiction entre sa morale de probité et le dogme. La sortie du christianisme n'est donc pas en tant que telle une bonne nouvelle, ni une nouvelle rassurante. Cependant, la leçon de Nietzsche est que l'avenir reste fondamentalement ouvert, y compris à une problématique « reviviscence du divin ».

2 La pensée de Nietzsche peut-elle être de quelque intérêt pour qui s'interroge sur l'avenir de la religion en ce début de troisième millénaire ? Si Nietzsche se réduisait à la vulgate avec laquelle on confond sa philosophie religieuse, à savoir la mort de Dieu et (la conjonction semble aller de soi) le triomphe d'un homme enfin libéré et autonome, délié qu'il serait de croyances infantiles et paralysantes, nous n'aurions guère à nous arrêter à sa pensée. Nous voyons trop bien perdurer les religions, même sous des formes inquiétantes et menaçantes, nous savons trop bien aussi à quel point le mythe d'un homme enfin libéré des illusions et adulte a volé en éclat pour que nous nous arrêtions longtemps à ces fausses prophéties. Mais justement si Nietzsche peut nous intéresser, c'est-à-dire en philosophe nous aider à penser à notre tour la situation religieuse originale qui est la nôtre, c'est que sa pensée ne s'identifie pas à ces lieux communs, même si des lectures rapides ou intéressées l'ont réduit à ces pauvretés, y compris du côté de théologiens chrétiens.

Il faut préciser immédiatement que Nietzsche s'interroge peu sur l'avenir de la religion en tant que telle, si l'on veut entendre par là qu'il parlerait de religion en général, ou mettrait en cause un soi-disant sentiment religieux pour en montrer la vanité, ou, projet encore plus improbable, qu'en philosophe des religions qu'il n'était pas, il prophétiserait sur le destin du religieux en soi. On doit d'ailleurs noter tout de suite qu'il doute si peu de la vivacité et de la permanence du sentiment religieux en l'homme qu'il définit l'homme comme "fabricateur de dieux», comme créateur fécond et inépuisable d'idoles, au point même, lit-on dans l'Avant-propos du Crépuscule des idoles qu'« il y a dans le monde plus d'idoles que de réalités ». Formule bien remarquable qui 
atteste à quel point l'idolâtrie en tant que fabrication d'idoles est sans doute une caractéristique de l'homme. Formule remarquable encore en ce qu'elle nous avertit d'emblée : quand bien même les religions seraient mortes ou affaiblies, et notamment le christianisme, rien n'indique pour autant que l'homme cessera d'être animé par « la volonté de croyance ", c'est-à-dire par le besoin de se donner des idoles, des certitudes inébranlables, des points d'appui fermes pour porter et supporter l'existence. Et il est clair pour Nietzsche que le monde moderne grouille de pareilles idoles qui ont nom "progrès ", "scientisme ", " avènement du bonheur pour tous ", "socialisme " ou "droits de l'homme », voire " athéisme » qui, si l'on en croit La Généalogie de la morale (III, § 27), ne serait que l'interprétation la plus subtile et la plus voilée de la volonté de vérité à tout prix, donc de la volonté de croyance... Ainsi la religion peut-elle parfaitement survivre au déclin de son emprise institutionnelle, dogmatique ou morale sur les esprits, sous la forme de l'idolâtrie moderne qui a fondamentalement selon Nietzsche les mêmes caractéristiques que les anciennes religions, avec en plus l'illusion de s'être libérée de la religion. Une religion sans religion, une croyance sans le masque du religieux, voilà de quoi enfermer le « tard venu » moderne ou "l'homme supérieur » qui se croit plus malin que les hommes du passé, dans des pièges qu'il aura bien du mal à éviter.

4 Ainsi convient-il de s'arrêter un peu sur le sort de la religion dans le monde moderne, et notamment sur le destin du christianisme. Nietzsche a trop souvent insisté sur le fait qu'il parlait à partir de lui-même et de l'expérience vive et douloureuse de sa propre éducation, de sa maladie, de son destin individuel, pour ne pas admettre qu'en fait de religion, il parle essentiellement du christianisme, du christianisme historique certes qui a marqué l'Europe, mais très précisément du piétisme luthérien qui l'a façonné pour le meilleur et pour le pire. Parlant de religion, c'est donc de christianisme pour l'essentiel que Nietzsche parle. Or, et telles sont les thèses que je vais avancer dans cette intervention, nous sommes «au lit de mort du christianisme » selon le titre de l'aphorisme 92 d'Aurore; mais cette " euthanasie » n'annonce rien de bon, elle laisse présager d'innombrables bouleversements qui peuvent précipiter la perte même de l'humanité ; car dans la mort du christianisme se joue le destin du nihilisme, donc nullement l'assurance d'un règne de la vérité, de la liberté, de l'homme enfin advenu à lui-même; du coup nous devrons nous interroger, quelle sera la ou les figures de la religion dans l'avenir?

Euthanasie du christianisme

5 Si le christianisme est frappé de mort, il faut bien voir qu'aux yeux de Nietzsche la cause n'est pas à chercher à l'extérieur du christianisme lui-même. Très différent en cela du rationalisme dominant en son siècle, et même de la philosophie des Lumières qu'il admira pourtant à l'époque de Humain trop humain, il ne pense pas que la religion reculerait devant les poussées d'une raison davantage maitresse d'elle-même, ou sous les « avancées » des sciences. Il affirme même dans l'aphorisme 300 du Gai Savoir que ce sont les religions qui ont donné une impulsion décisive à la soif de connaissance d'où sont nés les sciences, car elles ont ouvert l'homme à un univers plus vaste que l'univers familier du monde sensible, et elles ont suscité en celui-ci « la faim et la soif de soi-même et à trouver en soi rassasiement et plénitude "; et de manière hautement significative le texte cite ensuite Prométhée comme caractéristique de cette illusion typiquement religieuse "d'avoir dérobé la lumière ", avant de découvrir que ce désir de lumière était « l'œuvre de ses mains ». Mais il n'en reste pas moins que l'impulsion à connaître est venue de la religion. De manière plus générale, s'il n'y a aucune « vérité » dans les 
religions, selon l'aphorisme 110 de Humain trop humain, la religion est utile, même nécessaire à l'homme en ce qu'elle lui donne la force de vivre ${ }^{1}$; elle n'est donc pas à mettre du côté des illusions en tous points néfastes, pas non plus du côté de ces erreurs dont on pourrait se passer facilement. Si la religion donne à l'homme la force de vivre et si la religion s'efface, où trouver cette force de vivre ? Est-il sûr même que l'homme moderne la trouvera facilement?

6 L'originalité de la critique nietzschéenne du christianisme vient de ce que Nietzsche considère le christianisme comme une religion essentiellement réactive, c'est-à-dire foncièrement instable et contradictoire. Il est bâti sur des contradictions qui ne peuvent pas ne pas s'effondrer à plus ou moins long terme, et ce terme est désormais arrivé. Si l'on suit les analyses de l'aphorisme 357 du Gai Savoir (et l'on pourrait s'appuyer sur d'autres textes qui conduiraient à des approches sensiblement différentes, mais cohérentes pour l'essentiel avec ce passage), on dira que le christianisme meurt de la contradiction entre sa morale et le dogme. Le croyant est en effet éduqué à l'examen de conscience, à la rigueur d'analyse de ses actes, à la minutie dans l'appréciation des mouvements de son âme par rapport à la volonté de Dieu. De ce point de vue le christianisme apporte une insistance sur la subjectivité qu'ignoraient les Grecs antiques, insistance telle que tout retour aux Grecs est désormais impossible, puisque nous ne parvenons même plus à comprendre leur univers moral et religieux. Mais cette éducation patiemment faite au cours des siècles avive l'honnêteté intellectuelle du croyant, et de manière général de l'Européen formé par le confessionnal et la direction de conscience. Vient un jour où la probité intellectuelle se retourne sur le système de croyance; celui-ci devient proprement incroyable (foi en une Providence menant l'histoire, en la bonté d'un Dieu qui dispose tout pour le meilleur, donc en l'idée d'une " finalité morale de l'ordre universel », en la Rédemption sacrificielle par le Christ, etc.). Ce qui s'oppose donc au christianisme, ce sont moins nos arguments que notre goût : nous ne pouvons plus, «nous bons Européens héritiers de cette selbstüberwindung la plus durable et la plus courageuse dont l'Europe ait fait preuve », entrer dans le système de croyance proposé par le christianisme. Celui-ci en tant qu'éducateur nous a aliéné à lui-même, et il s'effondre du dedans par une lente érosion dont la Réforme luthérienne, par exemple, fut un moment essentiel et typiquement réactif (Luther croyait redonner vigueur primitive à la foi, mais il contribuait en réalité à sa ruine en désacralisant et le prêtre et la Bible livrée à l'emprise de chacun).

7 On pourrait dire en d'autres termes que le christianisme a survalorisé l'homme, l'a mis au centre, lui a fait croire en sa valeur infinie auprès de Dieu au point qu'il était nécessaire que Dieu acceptât le sacrifice de son propre Fils pour sa Rédemption (selon la version paulinienne du christianisme que critique particulièrement Nietzsche). Il importe au plus haut point de bien noter l'accusation centrale, généralement méconnue des interprètes, particulièrement des théologiens chrétiens: elle porte contre cet anthropocentrisme chrétien, contre cette boursouflure orgueilleuse de l'homme à qui l'on fait croire qu'il est plus important qu'il n'est en réalité, puisque, selon les principes essentiels du christianisme, Dieu se tourne vers lui et a souci de son salut. Anthropomorphisme proprement risible, puisque l'homme n'est qu'une fourmi perdue dans le vaste monde, Nietzsche n'a cessé de le répéter dans des textes fulgurants par leur implacable mise en cause de la superbe humaine trop humaine. C'est le christianisme qui, selon Nietzsche, donne une place excessive à l'homme, qui fait sortir l'existence de ses gonds et donc lui donne une importance qu'il n'a pas en 
réalité. Une telle «présomption » a d'ailleurs pour effet de précipiter dans une morale ascétique impitoyable, puisque le chrétien doit être à hauteur d'une vocation si haute et éliminer de sa vie toute attache passionnelle indigne de lui. Mais il a aussi pour effet à plus long terme de faire prendre conscience à l'homme de sa propre valeur unique. Par contrecoup il fait découvrir que le Dieu chrétien trop bon et trop miséricordieux n'a qu'un visage trop humain, qu'il est l'idéal tout humain sous couvert duquel l'homme s'affirmait et se valorisait; du coup ce Dieu ne peut plus être reconnu que comme un dieu pitoyable, non comme le divin même. C'est cette logique autodestructrice qui a engendré l'athéisme moderne, c'est donc le Dieu chrétien qui meurt par inconsistance, contradiction et exténuation de soi. En ce sens l'athéisme est le fruit du christianisme lui-même, manifestant au grand jour son essence destructrice et nihiliste.

Sombre avenir

8 La «mort de Dieu » n'est donc pas provoquée de l'extérieur du christianisme, mais par le christianisme lui-même. C'est d'ailleurs si vrai que dans le texte célèbre du Gai Savoir (§125) où l'exalté annonce cette mort sur la place publique les athées qui la peuplent n'en croient pas leurs oreilles et se rient de cette annonce, donc ne se reconnaissent pas en elle; c'est d'ailleurs dans les églises finalement que l'exalté va délivrer son message... Il est par conséquent notable que les athées ne se rendent pas compte de la portée de l'événement, qu'ils n'en mesurent pas les dimensions totales. En un sens cette mort s'est déjà produite (le Dieu chrétien est devenu incroyable), mais comme des étoiles mortes continuent à rayonner pendant des siècles et à éclairer nos nuits, cette mort n'a pas encore produit ses effets. On soupçonne à peine sa portée. « Le plus grand événement récent - à savoir que "Dieu est mort ", que la croyance au Dieu chrétien est tombée en discrédit - commence dès maintenant à étendre son ombre sur l'Europe ", lit-on au début de l'aphorisme 343 du Gai Savoir. Mais c'est une ombre qui se répand, non point une lumière ou une aurore nouvelle. Ombre menaçante qui risque de faire disparaître systèmes de croyances, valeurs et institutions des Européens, "éclipse de soleil comme jamais il ne s'en produisit en ce monde ». Tout par conséquent, sauf le règne des Lumières... Du moins "nous autres devineurs d'énigmes", "placés entre aujourd'hui et demain », « devrions être capables de discerner les ombres sur le point de recouvrir l'Europe». Mais qui pourra prophétiser «cette formidable logique de terreurs » et de déstabilisation qui fera perdre à la terre humaine son équilibre? Comme le clamait l'exalté, "qu'avons-nous fait à désenchaîner cette terre de son soleil ? Vers où roule-t-elle à présent? Ne sommes-nous pas précipités dans une chute continue? N'errons-nous pas comme à travers un néant infini? Ne sentons-nous pas le souffle du vide? Ne fait-il pas nuit sans cesse et de plus en plus nuit? ». Car « sitôt que nous rejetons loin de nous l'interprétation chrétienne, que nous condamnons son "sens" comme de la fausse monnaie, la question schopenhauerienne nous assaille de la manière la plus terrible : l'existence a-t-elle seulement un sens? - question qui aura besoin de quelques siècles pour être entendue dans toute sa profondeur $"^{2}$.

9 Si la mort de Dieu n'ouvre pas de soi un univers de paix, de liberté et d'harmonie ou ne débouche pas sur un sens de l'homme « autonome » et sûr de lui-même, la raison en est que cette croyance était à la fois utile et nécessaire, et que faute de pouvoir assumer lucidement cette perte les "hommes supérieurs ", c'est-à-dire les modernes qui se croient tels, risquent bien de se livrer à toutes sortes d'idoles. Cette croyance était utile parce qu'elle permettait à l'homme de voiler sa détresse dans l'univers, de lui conférer une importance et une centralité qu'il n'a pas; une fois détruite ou compromise, la 
réalité de la condition humaine éclate avec force, mais cette réalité est insoutenable. Dans l'illustration de ce thème, les textes et les images abondent ; l'homme est comparé à une minuscule fourmi perdue dans une vaste forêt, ou à une mouche qui se prend pour le centre du monde alors qu'elle n'est qu'une réalité inconsistante, une poussière perdue dans l'immensité de l'univers. Or fondamentalement l'homme ne veut pas voir sa situation dans le chaos du monde; le caractère transitoire de l'individu redevient plus que jamais une épreuve qu'on tente d'annuler en se donnant de nouvelles croyances. On s'imaginera qu'on trouve sens à sa vie et sublimité à son existence à travailler au progrès des sciences, à l'avènement d'une société prospère et heureuse, au sens de l'histoire, à moins qu'on ne préfère « adorer le point d'interrogation » comme le dit ironiquement un passage de La Généalogie de la morale. La consolation religieuse cachait que la vérité est redoutable, cruelle, horrible à regarder en face, que «la connaissance est tourment» selon le titre de l'aphorisme 109 du premier livre de Humain trop humain. En d'autres termes, la vie est effrayante, inconnue, inconnaissable, impitoyable, ou, si l'on parvient à sortir des jugements valorisants-dévalorisants, on dira qu'elle est en elle-même innocente, donc par-delà bien et mal, par-delà sens et non-sens. La réalité de la vie ou du monde ne fait pas sens, mais n'est pas non plus insensée. Elle est ce qu'elle est, rien de plus. Il faudrait pouvoir l'affirmer et la vouloir telle quelle est, mais quelle puissance de volonté ne faudrait-il pas pour être capable d'un tel dire-oui?

10 Tout ceci conduit à comprendre que le véritable enjeu de cette crise majeure a nom «nihilisme». Le désir religieux lui-même comme l'aspiration métaphysique à un monde vrai a la même source : la volonté de substituer au monde sensible troublant, fugitif, incertain, un monde de stabilité, de vérité, de beauté, donc d'échanger le devenir pour l'Être. La même aspiration était à l'œuvre dans les religions et dans les métaphysiques, et sans doute faut-il souligner qu'aux yeux de Nietzsche, ainsi qu'on l'a vu plus haut à propos de la science, « le besoin métaphysique n'est pas, comme le veut Schopenhauer, l'origine des religions, mais un rejeton tardif de ces dernières. Sous l'empire des pensées religieuses, on s'est habitué à la représentation d'un "autre monde" (arrière-monde, inférieur, supérieur) si bien que la disparition du délire religieux fait éprouver une privation, un vide inquiétants " ${ }^{3}$. Or cette recherche de valeurs les plus hautes est animée par une volonté de néant, par le néant même, c'est-àdire par le refus de ce qui est. Et voilà bien en quoi consiste le nihilisme : substituer un monde fictif au monde réel, vouloir nier ce qui est au profit de ce qui n'est pas (néant). Or, pour les raisons déjà indiquées, mais aussi par suite de la critique du dualisme métaphysique qui fut une longue histoire (retracée à grands traits au $§ 357$ du Gai Savoir) et que je ne retiens pas ici, nous assistons à la dévalorisation des valeurs les plus hautes. Autre définition du nihilisme moderne: nous voyons sous les Idéaux à majuscule le néant qui les habite, comme nous voyons sous le Dieu chrétien la recherche toute humaine de se donner une divinité protectrice et pourvoyeuse de sens pour l'insignifiante poussière qu'est l'homme.

11 L'histoire n'avance donc pas sur une trajectoire réconfortante, et la sortie de la religion n'a rien de rassurant, parce qu'elle permet de découvrir l'emprise universelle du nihilisme, emprise existant depuis toujours en un sens, mais devenue plus évidente aujourd'hui. Un tel nihilisme lui-même ouvre à plusieurs possibilités : il peut conduire aux effondrements les plus tragiques, par désespérance, règne du non-sens, goût de la mort à travers drogue, suicide, anarchie destructrice. Il peut déboucher sur de nouvelles volontés de croyance d'autant plus impitoyables et inflexibles qu'elles seront 
la construction de volontés faibles et désireuses de s'identifier à de nouveaux esclavages. Mais le nihilisme peut être aussi le terrain d'un nihilisme positif, celui qui permettrait l'affirmation admirative de ce qui est tel que c'est, et qui pourrait ainsi bénir et chanter le monde. Ainsi l'avenir reste-t-il largement ouvert, tendu qu'il est entre des possibilités redoutables marquées par des effondrements complets de systèmes de valeurs ou d'institutions politiques (l'État en particulier dont les bases sont en réalité de nature religieuse, selon le paragraphe 472 de Humain trop humain et l'avènement d'un homme enfin capable de dire et de faire oui, ce que Ainsi parlait Zarathoustra appelle le surhomme.

12 Cet avenir est d'ailleurs si ouvert que Nietzsche annonce aussi qu'il pourrait être le lieu d'une "reviviscence du divin", d'une efflorescence d'un dire-oui sacré à l'éternité, donc d'une religion affirmative, non sous la forme d'institutions réglées ou d'Églises, cela va de soi, mais comme possibilité pour une volonté métamorphosée de chanter le chant de minuit et de l'éternité. Nietzsche lui-même confesse dans un Posthume de $1888{ }^{4}$ que, comme Zarathoustra, il ne croira qu'à un Dieu qui saurait danser, Dieu léger, disparaissant, non obsédant, ne promettant aucun salut éternel à la poussière humaine transitoire, Dieu visiteur, tel le Dionysos du dernier aphorisme de Par-delà bien et mal. Dieu auquel on ne croira pas au sens où on l'investirait d'une volonté de croyance, mais qu'on chantera et bénira grâce à une distance maintenue ou à un respect qui refuse toute forme d'appropriation ou de captation. Mais il faut pouvoir s'émanciper des religions ascétiques (notamment des religions de salut) pour entrevoir le sens de ce qu'il faut bien appeler une "mystique", en mettant des guillemets pour ne pas effaroucher les tenants de la Vulgate confortable dont j'ai parlé en commençant. Or, constate un aphorisme de Humain trop humain ${ }^{5}$, « il n'y a pas assez de religion dans le monde pour seulement anéantir toute religion». Seul un surcroît de sens du divin pourrait donc anéantir ces religions qui ont tué en l'homme le goût du divin. Ainsi, selon un texte posthume des années 1880-1881, « ce n'est qu'après la mort de la religion que l'intervention $d u$ divin pourra reprendre toute sa luxuriance " ${ }^{6}$. Divin sauvage, réalité abyssale, nouvel infini, autant de termes à prendre en considération en évitant de les chosifier, mais qui tous indiquent bien que l'athéisme nietzschéen n'est paradoxalement nullement antireligieux, mais de nature mystique (si l'on emploie à nouveau ce terme avec la prudence voulue). Impossible en tout cas de lire Ainsi parlait Zarathoustra sans sentir la ferveur mystique qui traverse ces pages, pour ne rien dire des autres poèmes de l'œuvre. Parfois même Nietzsche laisse entendre que ce divin foisonnant pourrait prendre la forme d'un nouveau polythéisme, une fois éteinte l'emprise du "monotono-théisme ", mais je ne suis pas sûr qu'il faille accorder trop de poids à ces textes. On peut les interpréter ainsi et donc leur donner toute leur place : après la domination d'un Dieu unique et d'une religion ascétique, la luxuriance d'un divin non personnel serait une possibilité multiforme et proliférante.

Mais comme nous savons, ce dire-oui suppose une telle métamorphose de la volonté et l'aptitude à « se tenir en équilibre sur des cordes légères, des possibilités, et même au bord des abîmes à danser encore ${ }^{7}{ }^{7}$ qu'il ne sera guère possible à la multitude. Tel serait un athéisme vrai, qui ne fixe pas le divin sur un nom ou une personne, sur une identité repérable et rassurante. Athéisme aristocratique très éloigné de l'athéisme probable du grand nombre, qui pour Nietzsche reste encore une volonté de croyance, la plus tenace et la plus inconsciente car toujours animée par « la volonté de vérité ». Mais par ailleurs pour le grand nombre qui a besoin de religion, survivront les anciennes croyances, pour d'autres encore Nietzsche envisage le règne d'un bouddhisme mou, 
c'est-à-dire d'une adhésion à une sagesse sans dogme, marquée par la fatigue et le désespoir de soi, le retrait du monde et l'épuisement de soi.

$\mathrm{Au}$ total ces pronostics ne sont certes pas dénués de pertinence. Me risquant pour finir à un jugement sur eux, je proposerai quelques considérations personnelles. D'abord Nietzsche est assurément allé un peu vite dans son annonce de la mort du christianisme ; cet effet d'annonce, comme nous dirions aujourd'hui, garde un aspect de provocation, mais il n'est pas non plus dénué de tout ressentiment, et ce ressentiment a conduit à un jugement assez aveugle. Certes, sa critique doit être entendue ; mais pour le fond il n'a guère vu ni l'extraordinaire aptitude à persévérer du christianisme, ni (encore moins) sa capacité à évoluer, à accepter le jeu de l'interprétation de ses croyances fondamentales ou de ses textes fondateurs. Trop marqué par une application simplificatrice d'une généalogie pourtant subtile selon sa propre théorie, Nietzsche a mal apprécié les possibilités de renouvellement, bien qu'elles se soient accompagnées de terribles déchirements et de remises en cause qui ont secoué fortement le système. Surtout sa lecture du christianisme reste trop marquée, et par le piétisme de son enfance, et par le libéralisme de ses années d'études à Bonn sous l'influence de l'exégète David F. Strauss, au point qu'il a en quelque sorte « inventé » un système, tout à fait caractéristique de sa propre pensée, mais étranger aux traditions chrétiennes en ce qu'elles ont eu de plus vivant et de plus fort sur le plan théologique ou spirituel.

Ensuite on peut tenir pour particulièrement intéressant son diagnostic d'une reviviscence du divin ; l'effacement de l'emprise des Églises ne signifie pas pour autant la mort de la recherche religieuse, nous le savons bien. Un religieux qu'on pourrait dire "sauvage ", hors contrôle institutionnel, hors domination morale, prolifère de nos jours, et paradoxalement Nietzsche l'athée ne fut pas aveugle au point de croire en la mort pure et simple de l'instinct religieux, comme il dit; au contraire il fut capable d'avancer l'idée, si scandaleuse que les interprétations dominantes de sa philosophie se gardent bien d'en parler, qu'après la disparition et l'affaiblissement des religions institutionnelles, un autre divin délié de "moraline " pourrait apparaître. Et en effet nous voyons bien que l'instinct religieux n'est pas mort, même s'il conduit à nombre d'aberrations individuelles ou collectives. En ce sens la philosophie nietzschéenne prédispose assez remarquablement à un regard éveillé sur les réalités religieuses présentes. Aussi hésitera-t-on à aligner Nietzsche sur les deux autres prétendus maîtres du soupçon, Marx et Freud, qui sur ce point avaient cru pouvoir affirmer avec assurance, soit la mort de l'attitude religieuse comme telle, soit la fin d'une illusion.

Elle oblige enfin (et ce point est essentiel) à ne pas minimiser l'emprise universelle du nihilisme. Cette emprise affecte profondément nos sociétés, ne serait-ce qu'à travers cette perte des repères fondamentaux qu'annonce l'exalté du Gai Savoir. Or l'effondrement de la morale faisait partie de ce « grand spectacle en cent actes, réservé aux deux prochains siècles" qu'annonçait La Généalogie de la morale, spectacle jugé comme "le plus redoutable et le plus problématique». C'est si vrai qu'à ce propos certains philosophes (J.-F. Mattei) ou sociologues (J.-P. Le Goff) ne craignent pas de parler de «barbarie » pour caractériser le temps présent, en ce sens que l'individu moderne et plus généralement nos sociétés sont incapables de se structurer autour de quelques valeurs fondamentales, de les intérioriser, et donc de dominer leur violence. Il serait donc possible d'analyser le désarroi de la modernité à travers l'emprise du nihilisme, en notant que les effets corrosifs et destructeurs de ce nihilisme ne cessent 
de concerner des sphères toujours plus vastes de l'existence humaine : religion, on l'a vu, politique, économique, affectivité, vie internationale, etc.

Pour s'en tenir à la sphère religieuse proprement dite, il est clair que le nihilisme actuel déstabilise les institutions religieuses; il suscite peurs et replis sur soi devant des réalités qu'on peine à comprendre ; il impose un style d'autorité qui au lieu d'aider des volontés à se construire les accable sous des impératifs moraux souvent intenables... D'où toutes sortes de distorsions et de maladies qui affectent les Églises. Par certains côtés les fondamentalismes et les intégrismes qui rongent les grandes religions (et pas seulement les religions monothéistes, il suffit de penser à l'hindouisme) sont des efforts désespérés pour combattre le nihilisme en croyant pouvoir en revenir à des bases fermes, incontestables, non minées par la fragilité, alors que c'est le mouvement de «retour » lui-même qui manifeste le désespoir et la peur qui l'anime. Et si Nietzsche parle peu du «mahométanisme » comme il dit, faute de connaissances et sans doute d'intérêt pour l'islam, il me semble que son analyse ne manque pas d'éclairer le destin des fondamentalismes qui marquent cette religion à peu près partout où elle domine et sous des formes réellement préoccupantes.

18 Si je devais conclure sur une note personnelle, je dirais que Nietzsche le provocateur oblige son lecteur à s'interroger sur lui-même et sur ses propres croyances, non point pour adopter celles du philosophe, car il s'est toujours refusé lui-même à faire école, mais pour se métamorphoser à l'écoute de l'analyse généalogique. Il met d'ailleurs en garde contre toute tentative d'adaptation à l'air du temps, sous prétexte de se survivre. Et il ne manque pas de pages extrêmement dures contre un christianisme accommodé, fade, qui a "glissé vers un doux moralisme", celui de la bienveillance universelle, animé par « la croyance que, dans tout l'univers, bienveillance et sens des bienséances finiront par dominer ${ }^{8}$. En fidélité à la généalogie, il convient de faire retour sur soi, de se demander ce qui doit être vraiment voulu et ce qui fait réellement sens contre les surcharges qui défigurent le message évangélique. Je retiens pour ma part la mise en garde envers une religion moralisée et coercitive qui obsède tellement l'homme de luimême qu'elle finit par lui faire perdre le sens du divin. Dès lors il s'agit de se demander si dans notre façon de vivre et de penser la foi chrétienne, nous savons assez lutter contre un anthropocentrisme qui rend l'homme prisonnier de ses peurs et avide de souffrir pour se démontrer à lui-même sa fidélité. Cela engage certes toute une conception de la morale, mais tout autant une christologie et le sens authentique d'une Rédemption qui ne soit pas perverti par une exaltation indue de la souffrance et du péché. Découvrir la gratuité du dire-oui et la beauté du geste d'abandon de soi, restaurer le goût du divin devrait être une exigence pour sortir le christianisme de ses peurs et des maladies qui ont tant contribué à sa dégradation et à sa perte de pertinence auprès de nombre de nos contemporains. Et si Nietzsche propose à son lecteur qu'il devienne davantage ce qu'il est, alors nous pourrions dire qu'à son contact, nous ne devenons pas moins chrétien, mais que nous le devenons autrement. Faut-il ajouter enfin que ce qui vient d'être suggéré pour le chrétien, peut être entendu de tout homme qui pourrait gagner à plus de noblesse et de respect envers la réalité tel qu'elle est, et d'abord envers lui-même, si toutefois il a des oreilles pour entendre quelque chose du message nietzschéen? Voilà autant de questions qui concernent le présent, mais qui peuvent aussi aider à construire cet avenir qui préoccupait tant le philosophe Nietzsche. 


\section{NOTES}

1.. Cf. aussi Le Gai Savoir $\$ 110$.

2.. Le Gai Savoir, § 357.

3.. Le Gai Savoir, § 151.

4.. Euvres philosophiques complètes, Paris, Gallimard, 1977, tome XIV, Fragments posthumes $17[4], \S 5$.

5.. Tome $1, \S 123$.

6.. La Volonté de puissance, Paris, Gallimard, coll. « Tel » 260, 1995, tome II, § 581.

7.. Le Gai Savoir, § 347.

8.. Aurore, § 92 .

\section{RÉSUMÉS}

Nietzsche, qui définit l'homme comme «fabricateur de dieux» est, avant tout, un critique de l'idolâtrie qui peut prendre bien d'autres formes que celle de la religion. Le christianisme est, selon lui, à l'origine de sa propre « euthanasie » qui résulte d'une contradiction entre sa morale de probité et le dogme. La sortie du christianisme n'est donc pas en tant que telle une bonne nouvelle, ni une nouvelle rassurante. Cependant, la leçon de Nietzsche est que l'avenir reste fondamentalement ouvert, y compris à une problématique « reviviscence du divin ». 